\title{
Nonlinear Analysis of a New Car-Following Model Based on Internet-Connected Vehicles
}

\author{
Lei $\mathrm{Yu}^{1 *}$, Bingchang Zhou ${ }^{2}$, Zhongke Shi ${ }^{1}$ \\ ${ }^{1}$ College of Automation, Northwestern Polytechnical University, Xi'an, Shaanxi Province, China. \\ ${ }^{2}$ School of Science, Northwestern Polytechnical University, Xi'an, Shaanxi Province, China. \\ * Corresponding author. Tel.: (+86)15339260284; email: yuleijk@126.com \\ Manuscript submitted July 9, 2014; accepted December 6, 2014. \\ doi: 10.17706/ijcce.2015.v4.390
}

\begin{abstract}
A new car-following model is proposed based on Internet-connected vehicles. In this model, a vehicle is controlled by the information of arbitrary number of vehicles obtained from the Internet-connected vehicles system. The stability condition is investigated by using the linear stability theory. The result shows that the stability of traffic flow is improved by taking into account the headway of vehicles ahead and the relative velocity. By applying the nonlinear analysis, the modified Korteweg-de Vries ( $m K d V$ ) equation is derived to describe the traffic jams. From the numerical simulation, it is shown that the traffic jams are suppressed efficiently by taking into account the headway of vehicles ahead and the relative velocity.
\end{abstract}

Key words: Traffic flow, Internet-connected vehicles, modified Korteweg-de Vries equation, Kink-antikink soliton, density wave.

\section{Introduction}

Traffic flow, especially of traffic jams, is an interesting problem. To understand the rich variety of vehicular traffic, many traffic flow models are proposed to describe traffic flow, such as car-following models, cellular automaton (CA) models, gas kinetic models and hydrodynamic models [1]-[19].

The advantage of car-following models is that we can easy analyze the analytical structure of the models. One car-following model is proposed and analyzed by Newell [20] and Whitham [21]. The optimal velocity (OV) model, another version of car-following model, is proposed by Bando et al [22] without introducing a delay time. The OV model successfully describes the dynamical formation of traffic jams and reveals the transition mechanism very simply. In the OV model, acceleration of vehicle is described by a simple differential equation using the optimal velocity function, which is depended on the headway (the distance between two successive vehicles), that is, the driver is supposed to look at the preceding car only. In more realistic situation, the driver considers more vehicles around him/her. From the viewpoint of control theory for traffic flow, that is important to suppress the formation of traffic congestion. So there have been many works to extending the OV model to more realistic traffic model [23]-[25].

The Internet-connected vehicles era comes based on the technologies of wireless network communication, GPS and other information technology. Vehicles can communicate with each other by vehicle to vehicle (V2V) communication and with roadside base stations by vehicle to infrastructure (V2I) communication in vehicular network [26]. Based on the fact that real-time systems of traffic information are becoming widely available, where each vehicle can receive information of other vehicles and then decide its 
optimal behavior, the studies have been focused on how to use the information owned to suppress the appearance of traffic congestion efficiently. Hasebe et al. [27] discuss the property of the forward looking OV model, in which the headway of arbitrary number of vehicles that precede are considered. Ge et al. [28]-[29] discuss the stability condition of the model which take into account the headway of arbitrary number of vehicles ahead and derive the modified Korteweg-de Vries (mKdV) equation and Korteweg-de Vries (KdV) equation respectively. Li and Liu [30] introduce relative velocity of arbitrary number of vehicles ahead into the OV model and find that the stability of traffic flow is improved.

The purpose of this paper is to analyze the Newell- Whitham-type car-following model which considers not only the headway of arbitrary number of vehicles ahead but also the relative velocity. We analyze the effect of the headway of vehicles ahead and the relative velocity upon the stability of traffic flow by using the linear theory. The result shows that the stability of traffic flow is improved by taking into account the headway of vehicles ahead and the relative velocity. Moreover, we apply the nonlinear analysis to derive the mKdV equation near the critical point and obtain its kink-antikink soliton solution to describe traffic jams. Finally we carry out the computer simulation for the extended model with periodic boundary condition. The numerical simulation is good agreement with the analytic results.

\section{Model}

Newell [20] and Whitham [21] propose a model which is given by a first-order differential equation by introducing a delay time which plays an important role in the occurrence of traffic congestion. The motion of $j$ th car is given as follows

$$
\frac{\mathrm{d} x_{j}(t+\tau)}{\mathrm{d} t}=V\left(\triangle x_{j}(t)\right),
$$

where $\Delta x_{j}(t)=x_{j+1}-x_{j}$ is the headway between two successive vehicles of the $j$ th vehicle at time $t$, $V\left(\triangle x_{j}(t)\right)$ is the optimal velocity function and $\tau$ is the delay time.

According to the idea mentioned above, we extend the Newell-Whitham-type model (1) to take into account the headway of $n$ - cars ahead and the relative velocity. Then the motion of $j$ th car is described by the following differential equation

$$
\frac{\mathrm{d} x_{j}(t+\tau)}{\mathrm{d} t}=V\left(\triangle x_{j}(t), \triangle x_{j+1}(t), \cdots, \triangle x_{j+n-1}(t), \triangle v_{j}(t)\right),
$$

where $v$ is the extended optimal velocity function including variables of the relative velocity $\nabla v_{j}(t)$ and the headway of $n$-cars ahead of the $\mathrm{j}$ th vehicle. We assume that the driver can obtain the information of $n$-vehicles that precede based on Internet-connected vehicles. We assume that the extended optimal velocity function is

$$
V\left(\triangle x_{j}(t), \triangle x_{j+1}(t), \cdots, \triangle x_{j+n-1}(t), \triangle v_{j}(t)\right)=V\left(\triangle x_{j}(t), \triangle x_{j+1}(t), \cdots, \triangle x_{j+n-1}(t)\right)+\lambda \triangle v_{j}(t),
$$

where the weighted coefficient of the relative velocity $\lambda$ is a constant independent of time, velocity and position $0 \leq \lambda \leq 1$. Then, the extended model (2) can be rewritten as

$$
\frac{\mathrm{d} x_{j}(t+\tau)}{\mathrm{d} t}=V\left(\Delta x_{j}(t), \Delta x_{j+1}(t), \cdots, \Delta x_{j+n-1}(t)\right)+\lambda \Delta v_{j}(t),
$$

If $\lambda=0$, Eq.(3) is the model which is proposed by Ge et al. [28]-[29].

We rewrite Eq.(3) to obtain the difference equation 


$$
x_{j}(t+2 \tau)-x_{j}(t+\tau)=\tau V\left(\triangle x_{j}(t), \triangle x_{j+1}(t), \cdots, \triangle x_{j+n-1}(t)\right)+\lambda\left(\triangle x_{j}(t+\tau)-\triangle x_{j}(t)\right) .
$$

The optimal velocity function is selected similar to that proposed by Bando et al. [22]

$$
V\left(\triangle x_{j}(t), \triangle x_{j+1}(t), \cdots, \triangle x_{j+n-1}(t)\right)=\frac{v_{\max }}{2}\left[\tanh \left(\sum_{l=0}^{n-1} \alpha_{l} \triangle x_{j+l}(t)-h_{c}\right)+\tanh \left(h_{c}\right)\right]
$$

where $v_{\text {max }}$ is the maximal velocity, $h_{c}$ is the safety distance and $\varepsilon$ is the weighted coefficient of $\nabla \chi_{j+i}(t)$. We suppose that $a_{i}$ have the following properties:

1) $a_{i}(l=0,1, \ldots n-1)$ decrease monotonically with increasing $l$, that is, $a_{i}<a_{i-1}$. The reason is that as the distance between the car ahead and the considered car increases the effect of cars ahead on the car motion reduces gradually.

2) $\sum_{l=0}^{n-1} \alpha_{l}=1, a_{i}=1$ for $n=1$. Here we select $a_{i}=6 / 7_{l+1(l \neq n-1)}$ and $a_{i}=6 / 7_{l+1(l=n-1)}$ for $n>1$.

We name $\sum_{l=0}^{n-1} \alpha_{l} \Delta x_{j+l}(t)$ in Eq. (5) as the weighted headway. The optimal velocity function $V$ in Eq. (5) is a monotonically increasing function of the weighted headway and has an upper bound (i.e. the maximal velocity). When the weighted headway is less than the safety distance, the vehicle reduces its velocity to prevent from crashing into the vehicle ahead. Otherwise, if the weighted headway is larger than the safety distance, the car velocity increases to the maximal velocity. The optimal velocity function $V$ has the turning point (inflection point) at $\sum_{l=0}^{n-1} \alpha_{l} \triangle x_{j+l}(t)=h_{c}$. It is important that the optimal velocity function $V$ has the turning point. Otherwise, we cannot derive mKdV equation which has the kink-antikink soliton solution to describe the traffic jams.

For later convenience, Eq. (4) can be rewritten in terms of the headway as follows

$$
\begin{gathered}
\triangle x_{j}(t+2 \tau)-\triangle x_{j}(t+\tau)=\tau\left[V\left(\sum_{l=0}^{n-1} \alpha_{l} \triangle x_{j+l+1}(t)\right)-V\left(\sum_{l=0}^{n-1} \alpha_{l} \triangle x_{j+l}(t)\right)\right] \\
+\lambda\left[\triangle x_{j+1}(t+\tau)-\triangle x_{j+1}(t)-\triangle x_{j}(t+\tau)+\Delta x_{j}(t)\right] .
\end{gathered}
$$

\section{Linear Stability Analysis}

We apply the linear stability theory to analyze the extended model described by Eq. (4). The stability of the uniform traffic flow is considered, which is such a state that all cars move with identical headway $h$ and optimal velocity $V(h, h \ldots h)$. The solution $\chi_{j}^{(0)}(t)$ representing the uniform steady state of Eq. (4) can be written as

$$
\chi_{j}^{(0)}(t)=h j+V(h, h \ldots h) t, h=\frac{L}{N}
$$

where $N$ is the total number of vehicles and $L$ is the road length.

Suppose $y_{j}(t)$ to be the small deviation from the uniform steady state $x_{j}^{(0)}(t): X_{j}(t)=X_{j}^{(0)}(t)+y_{j}(t)$. Substituting it into Eq.(6) and linearizing the resulting equation, we can obtain

$$
\triangle y_{j}(t+2 \tau)-\triangle y_{j}(t+\tau)=\tau V^{\prime}\left[\sum_{l=0}^{n-1} \alpha_{l}\left(\Delta y_{j+l+1}(t)-\Delta y_{j+l}(t)\right)\right]+\lambda\left[\Delta y_{j+1}(t+\tau)-\triangle y_{j+1}(t)-\triangle y_{j}(t+\tau)+\Delta y_{j}(t)\right]
$$


where $\Delta y_{j}(\mathrm{t})=y_{j+1}(t)-y_{j}(\mathrm{t})$. For simplicity, $V^{\prime}$ indicates the derivative of the optimal velocity function $V\left(\Delta x_{j}(t), \Delta x_{j+1}(t), \ldots \Delta x_{j+N-1}(t)\right.$ at $\Delta x_{j}(\mathrm{t})=\Delta x_{j+1}(t)=\cdots=\Delta x_{j+n-1}(t)=h \quad$ in the above equation and hereafter. By expanding, $\mathrm{y}_{j}(t) \propto \exp (\mathrm{i} k j+z t)$ the following equation of $z$ is obtained

$$
\left(\mathrm{e}^{z \tau}-1\right)\left[\mathrm{e}^{z \tau}-\lambda\left(\mathrm{e}^{\mathrm{i} k}-1\right)\right]=\tau V^{\prime}\left(\mathrm{e}^{\mathrm{i} k}-1\right)\left(\sum_{l=0}^{n-1} \alpha_{l} \mathrm{e}^{\mathrm{i} k l}\right) .
$$

By expanding $Z=z_{1}(i k)+z_{2}(\mathrm{i} k)^{2}+\cdots$ and inserting it into Eq.(9), the first- and second-order terms of $\mathrm{i} k$ are obtained

$$
z_{1}=V^{\prime}(h), z_{2}=-\frac{3}{2} \tau\left(V^{\prime}(h)\right)^{2}+\frac{V^{\prime}(h)}{2}\left(\sum_{l=0}^{n-1} \alpha_{l}(2 l+1)\right)+\lambda V^{\prime}(h)
$$

If $Z_{2}$ is a negative value, the uniform steady state is unstable for long wavelength modes, while the uniform flow is stable when $Z_{2}$ is a positive value. The neutral stability condition for the uniform steady state is given by

$$
\tau_{s}=\frac{2 \lambda+\sum_{l=0}^{n-1} \alpha_{l}(2 l+1)}{3 V^{\prime}(h)} .
$$

For small disturbances of long wavelength, the uniform traffic flow is stable if

$$
\tau<\frac{2 \lambda+\sum_{l=0}^{n-1} \alpha_{l}(2 l+1)}{3 V^{\prime}(h)} .
$$

The neutral stability line in the parameter space $(\Delta x, a)(a=1 / \tau)$ is shown in Fig. 1 for the extended model which is expressed by Eq.(4) where the word "CC" represents the coexisting curve and the word "SL" represents the spinodal line in the legend. From Fig. 1(a), it can be seen that the spinodal lines are lowered with taking into account larger value of $\lambda$ with same $n$. For $n=3$ and 5 with different $\lambda$, the curves related to the neutral stability lines and the coexisting curves are almost coincided in Fig. 1(b) and (c). It demonstrates that considering three cars ahead for $\lambda=0$ (i.e. $n=3$ ) is enough for the driver which is consistent with Ref. [28]. So considering two cars ahead for $\lambda=0.1$ (i.e. $n=2$ ) is enough for the driver from Fig. 1. In fact, these number of cars are closely related to the selection of $\alpha_{l}(l=0,1 \ldots n-1)$ and $\lambda$, that is, the weighted coefficients of the headway and the relative velocity. With different weight coefficients, a slightly different result will be obtained.
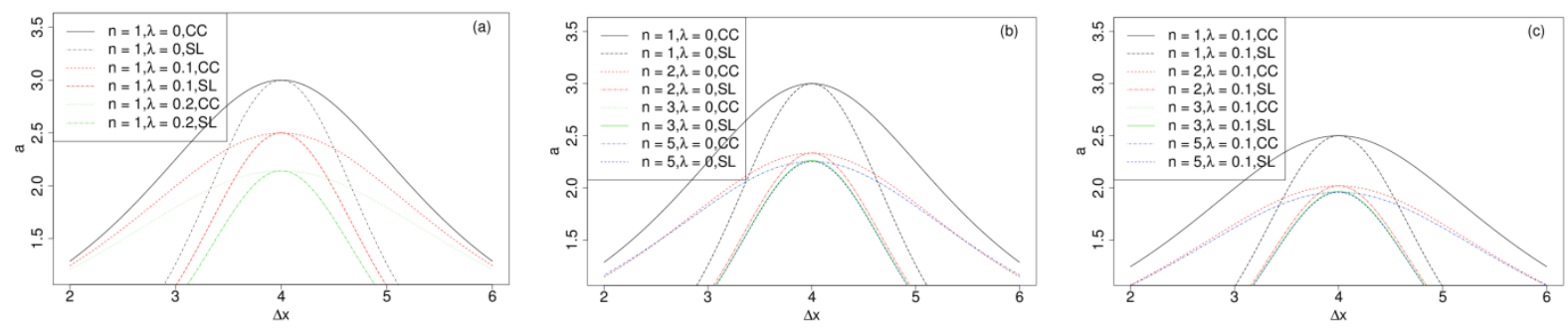

Fig. 1. The phase diagram in the parameter space $(\triangle x, a)$. 
From Fig. 1 it can be seen that the critical points and the neutral stability lines are lowered with taking into account more vehicles ahead and larger value of $\lambda$, which means the stability of the uniform traffic flow have been strengthened. The traffic jams are thus suppressed efficiently.

\section{Nonlinear Analysis}

The reductive perturbation method is applied to Eq. (6). We introduce slow scales for space variable $j$ and time variable $t$ and define slow variables $X$ and $T$ for $0<\varepsilon \ll 1$ as (see Ref. [31])

$$
X=\varepsilon(j+b t), T=\varepsilon^{3} t,
$$

where $b$ is a constant to be determined. We set the headway as

$$
\triangle x_{j}(t)=h_{c}+\varepsilon R(X, T) .
$$

Substituting Eqs. (13) and (14) into Eq. (6) and making the Taylor expansions about $\varepsilon$, we obtain the nonlinear partial differential equation given by Eq. (15)

$$
\begin{aligned}
\varepsilon^{2}\left(b \tau-V^{\prime} \tau\right) \partial_{X} R+\varepsilon^{3}\left[\frac{3}{2} b^{2} \tau^{2}-V^{\prime} \tau \sum_{l=0}^{n-1} \alpha_{l} \frac{2 l+1}{2}-\lambda b \tau\right] \partial_{X}^{2} R \\
+\varepsilon^{4}\left[\tau \partial_{T} R-\frac{V^{\prime \prime \prime}}{6} \tau \partial_{X} R^{3}+\left(\frac{7}{6} b^{3} \tau^{3}-V^{\prime} \tau \sum_{l=0}^{n-1} \alpha_{l} \frac{(l+1)^{3}-l^{3}}{6}-\lambda \frac{(1+b \tau)^{3}-(b \tau)^{3}-1}{6}\right) \partial_{X}^{3} R\right] \\
+\varepsilon^{5}\left[-\frac{V^{\prime \prime \prime} \tau}{6} \sum_{l=0}^{n-1} \alpha_{l} \frac{2 l+1}{2} \partial_{X}^{2} R^{3}+\left(3 b \tau^{2}-\lambda \tau\right) \partial_{X} \partial_{T} R+\left(\frac{5}{8} b^{4} \tau^{4}-V^{\prime} \tau \sum_{l=0}^{n-1} \alpha_{l} \frac{(l+1)^{4}-l^{4}}{24}\right.\right. \\
\left.+\left(3 b \tau^{2}-\lambda \tau\right) \partial_{X} \partial_{T} R+\left(\frac{5}{8} b^{4} \tau^{4}-V^{\prime} \tau \sum_{l=0}^{n-1} \alpha_{l} \frac{(l+1)^{4}-l^{4}}{24}-\lambda \frac{(1+b \tau)^{4}-(b \tau)^{4}-1}{24}\right) \partial_{X}^{4} R\right]=0,
\end{aligned}
$$

where $\quad V^{\prime}\left(h_{c}\right)=\left.\frac{\mathrm{d} V\left(\Delta x_{j}\right)}{\mathrm{d} \Delta x_{j}}\right|_{\Delta x_{j}=h_{c}}, V^{\prime \prime \prime}\left(h_{c}\right)=\left.\frac{\mathrm{d}^{3} V\left(\Delta x_{j}\right)}{\mathrm{d} \Delta x_{j}^{3}}\right|_{\Delta x_{j}=h_{c}}$. For simplicity, $V^{\prime}$ and $V^{\prime \prime \prime}$ correspond to $V^{\prime}\left(h_{c}\right)$ and $V^{\prime \prime \prime}\left(h_{c}\right)$ in the above equation and hereafter.

Near the critical point, $\tau=\left(1+\varepsilon^{2}\right) \tau_{c}$ and taking $b=V^{\prime}$, the second- and third-order terms of $\varepsilon$ are eliminated from Eq. (15). Then Eq. (15) can be rewritten as the simplified equation

$$
\partial_{T} R-g_{1} \partial_{X}^{3} R+g_{2} \partial_{X} R^{3}+\varepsilon\left[g_{3} \partial_{X}^{2} R+g_{4} \partial_{X}^{2} R^{3}+g_{5} \partial_{X}^{4} R\right]=0
$$

where

$$
\begin{aligned}
& \bar{\lambda}=2 \lambda+\sum_{l=0}^{n-1} \alpha_{l}(2 l+1), g_{1}=\frac{V^{\prime}}{54}\left[9 \sum_{l=0}^{n-1} \alpha_{l}\left((l+1)^{3}-l^{3}\right)+\lambda(27+9 \bar{\lambda})-7 \bar{\lambda}^{2}\right], g_{2}=-\frac{V^{\prime \prime \prime}}{6}, g_{3}=\frac{\bar{\lambda}}{2} V^{\prime}, g_{4}=\frac{\bar{\lambda}}{12} V^{\prime \prime \prime}, \\
& g_{5}=\frac{V^{\prime}}{216}\left[5 \bar{\lambda}^{3}-9 \sum_{l=0}^{n-1} \alpha_{l}\left((l+1)^{4}-l^{4}\right)-2 \lambda\left(2 \bar{\lambda}^{2}+9 \bar{\lambda}+18\right)\right]-\frac{V^{\prime}}{54}(\bar{\lambda}-\lambda)\left[7 \bar{\lambda}^{2}-9 \sum_{l=0}^{n-1} \alpha_{l}\left((l+1)^{3}-l^{3}\right)-9 \lambda(3+\bar{\lambda})\right] .
\end{aligned}
$$

In order to derive the standard mKdV equation with higher order correction, we can do the same procedure as Ref. [25]. The propagation velocity $A$ for the kink-antikink solution which is the solution of $\mathrm{mKdV}$ equation is as follows 


$$
A=\frac{5 g_{2} g_{3}}{2 g_{2} g_{5}-3 g_{1} g_{4}}
$$

Substituting the values $g_{1}-g_{5}$ into Eq. (17), we can obtain the value of propagation velocity for any vehicle that precede. The solution of the $\mathrm{mKdV}$ equation is obtained

$$
R(X, T)=\sqrt{\frac{g_{1} A}{g_{2}}} \tanh \left[\sqrt{\frac{A}{2}}\left(X-A g_{1} T\right)\right] .
$$

\section{Numerical simulation}

To check the validity of our theoretical results above, we carry out numerical simulation for the model described by Eq. (4) and (5) under the periodic boundary condition. First, we consider the impact of local small disturbances on the whole system. The vehicles move with the constant headway $h=4.0$. The initial conditions are chosen as follows

$$
\begin{aligned}
& \triangle x_{j}(0)=\triangle x_{j}(1)=4.0, \quad(j \neq 50,51) \\
& \triangle x_{j}(0)=\triangle x_{j}(1)=4.0-0.5, \quad(j=50) \\
& \triangle x_{j}(0)=\triangle x_{j}(1)=4.0+0.5, \quad(j=51)
\end{aligned}
$$

where the total number of cars is $N=100$, the safety distance is $h_{c}=4.0, v_{\max }=2, a=2.26$.

Fig. 2 shows the space-time evolution of the headway after a sufficiently long time $t=10^{4}$ for the extended model described by Eq. (4) and (5). The space-time evolution of the headway for $\lambda=0$ and $n=1,2,3,5$ are exhibited by the patterns (a), (b), (d) and (d). For $\lambda=0.1$ and $n=1,2,3,5$, those are exhibited by the patterns (c), (d), (d) and (d). The pattern (d) also exhibits the space-time evolution of the headway for $\lambda=0.2$ and $n=1$. In patterns (a), (b) and (c), the traffic flow is unstable because the stability condition (12) is not satisfied. The patterns (a) and (c) exhibit the coexisting phase in which the kink-antikink density waves appear as traffic jams and the density waves propagate backwards. That is to say, when small disturbances are added to the uniform traffic flow, the disturbances are amplified with time and the uniform flow changes finally to inhomogeneous traffic flow. The pattern (d) exhibits the freely moving phase after a sufficiently large time, that is, the traffic flow are stable with the same sensitivity.

The above analyses show that considering two cars that precede are enough for suppressing the traffic jams quickly and efficiently when $\lambda=0.1$ or three cars when $\lambda=0$. And one car is enough when $\lambda=0.2$. The influence of cars ahead is almost invariant after $n=2$ when $\lambda=0.1$ or $n=3$ when $\lambda=0$, which is consistent with the results in Fig. 1.

Fig. 3 shows the headway profile obtained at sufficiently large time $t=10298$ correspond to Fig. 2 . From Fig. 3, we find that the amplitude of the density wave decreases as the considered number of cars ahead and the value of $\lambda$ increases. In pattern (d) the density waves disappear and traffic flow is uniform over the whole space.

\section{Summary}

An extended car-following model is proposed by introducing the headway of arbitrary number of cars and the relative velocity for suppressing the traffic jams based on Internet-connected vehicles. The extended model has been analyzed by using the linear stability theory and the reductive perturbation method. The stability condition of the extended model is obtained and the results show that the stability of traffic flow is improved by taking into account not only the headway of arbitrary number of cars ahead but also the relative velocity. The kink-antikink soliton, solution of the $\mathrm{mKdV}$ equation near the critical point, is 
derived to describe the traffic jams. From the numerical simulation, the kink-antikink soliton is found. The simulation results confirm the linear stability analysis for the extended model and give the optimal state as $n=3(\lambda=0), n=2(\lambda=0.1)$ and $n=1(\lambda=0.2)$, that is, considering both the headway of cars ahead and the relative velocity are necessary to suppress the appearance of traffic jams efficiently based on Internet-connected vehicles. From the theoretical analysis and the numerical simulation, we can conclude that the traffic jams are suppressed efficiently by taking into account not only the headway of arbitrary number of cars ahead but also the relative velocity. The theoretical results are in good agreement with the simulation results.
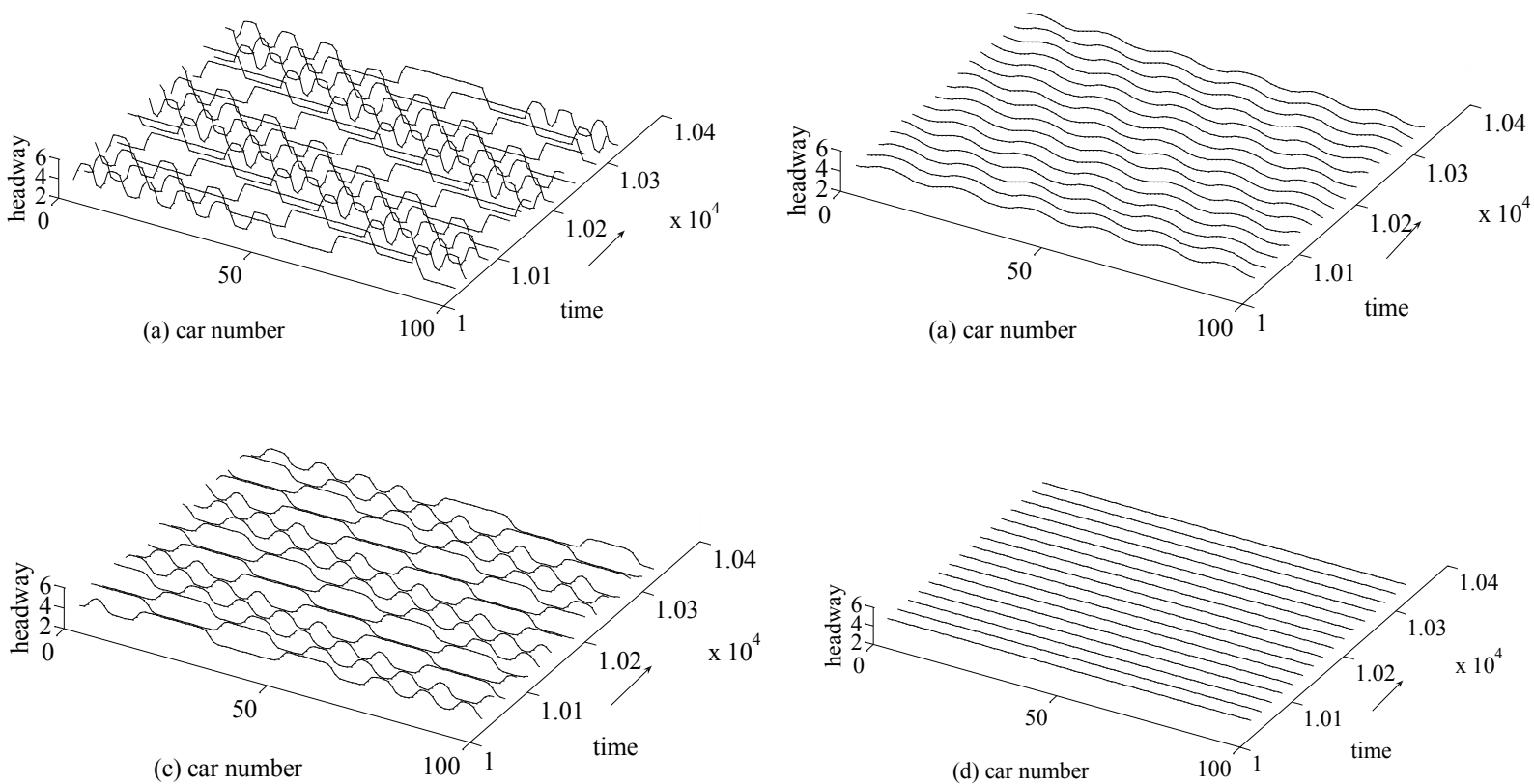

Fig. 2. Space-time evolution of the headway after $t=10,000$ for small disturbances.
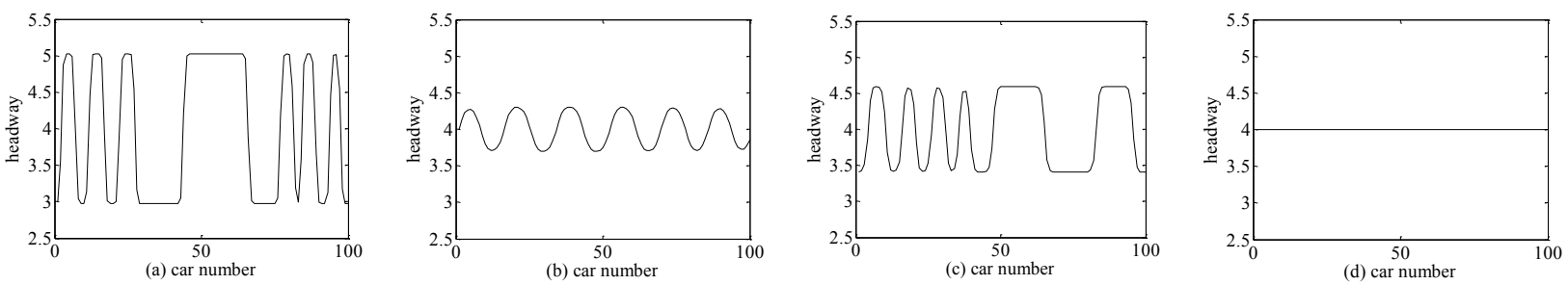

Fig. 3. Headway profile of the density wave at $\mathrm{t}=10298$ correspond to Fig. 2.

\section{Acknowledgment}

This work is supported by National Natural Science Foundation of China (Grant No.11102155, 11102165), Natural Science Foundation of Shaanxi Province (2013J Q7014), the new direction of the Young Scholar of Northwestern Polytechnical University and Foundation for Fundamental Research of Northwestern Polytechnical University (JC20110265).

\section{References}

[1] Schreckenberg, M., \& Wolf, D. E., editors. (1998). Traffic and granular flow'97. Springer.

[2] Helbing, D., Herrman, H. J., Schreckenberg, M., \& Wolf, D. E., editors. (2000). Traffic and Granular 
Flow'99. Springer.

[3] Chowdhury, D., Santen, L., \& Schadschneider, A. (2000). Statistical physics of vehicular traffic and some related system. Phys Rep, 329, 199-329.

[4] Helbing, D. (2001). Traffic and related self-driven many-particle systems. Rev Mod Phys, 73, 1067-1141.

[5] Nagatani, T. (2002). The physics of traffic jams. Rep Pro Phys, 65(9), 1331-1386.

[6] Ben-Naim, E., Krapivsky, P. L., \& Redner, S. (1994). Kinetics of clustering in traffic flows. Phys Rev E, 50, 822-829.

[7] Kurtze, D. A., \& Hong, D. C. (1995). Traffic jams, granular flow, and soliton selection. Phys Rev E, 52, 218-221.

[8] Feng, S. W., Gu G. Q., \& Dai, S. Q. (1997). Effects of traffic lights on CA traffic model. Commun Nonlinear Sci Numer Simul, 2, 70-74.

[9] Tomer, E., Safonov, L., Havlin, S. (2000). Presence of many stable nonhomogeneous states in an inertial car-following model. Phys Rev Lett, 84, 382-385.

[10] Treiber, M., Hennecke A., \& Helbing, D. (2000). Congested traffic states in empirical observations and microscopic simulations. Phys Rev E, 62, 1805-1824.

[11] Zhou, X. J., Liu Z. Z., \& Luo, J. (2002). The density wave in a car-following model. J Phys A, 35, 4495-4500.

[12] Gupta A. K., \& Katiyar, V. K. (2005). Analyses of shock waves and jams in traffic flow. J Phys A, 38, 4069-83.

[13] Lubashevsky, I., Kalenkov, S., \& Mahnke, R. (2002). Towards a variational principle for motivated vehicle motion. Phys Rev E, 65, 036140-1-5.

[14] Hu, Y. T. (1999). A new cellular automaton model for traffic flow. Commun Nonlinear Sci Numer Simul, 4 , 264-267.

[15] Maniccam, S. (2003). Traffic jamming on hexagonal lattice. Physica A, 321, 653-664.

[16] O'loan, O. J., Evans, M. R., \& Cates, M. E. (1998). Jamming transition in a homogeneous one-dimensional system: The bus route model. Phys Rev E, 58, 1404-1418.

[17] Huijberts, H. J. C. (2002). Analysis of a continuous car-following model for a bus route: existence, stability and bifurcations of synchronous motions. Physica A, 308, 489-517.

[18] Brockfeld, E., Barlovic, R., Schadschneider A., \& Schreckenberg, M. (2001). Optimizing traffic lights in a cellular automaton model for city traffic. Phys Rev E, 64, 056132-1-12.

[19] Kurata S., \& Nagatani, T. (2003). Spatio-temporal dynamics of jams in two-lane traffic flow with a blockage. Physica A, 318, 537-550.

[20] Newell, G. F. (1961). Nonlinear effects in the dynamics of car following. Oper Res, 9, 209-229.

[21] Whitham, B. G. (1990). Exact solutions for a discrete system arising in traffic flow. Proc $R$ Soc A, 428, 49-64.

[22] Bando, M., Hasebe, K., Nakayama, A., Shibata A., \& Sugiyama, Y. (1995). Dynamical model of traffic congestion and numerical simulation. Phys Rev E, 51, 1035-1042.

[23] Helbing, D., \& Tilch, B. (1998). Generalized force model of traffic dynamics. Phys Rev E, 53, 133-138.

[24] Jiang, R., Wu, Q. S., \& Zhu, Z. J. (2001). Full velocity difference model for a car-following theory. Phys Rev E, 64, 017101-1-4.

[25] Yu, L., Shi Z. K., \& Li, T. (2014). A new car-following model with two delays. Physics Letters A, 378, 348-357.

[26] Dimitrakopoulos, G. (2011). Intelligent transportation systems based on Internet-connected vehicles: fundamental research areas and challenges. Proceedings of the 11th Int. Conf. ITS Telecommunications (pp. 145-151).

[27] Hasebe, K., Nakayama A., \& Sugiyama, Y. (2004). Equivalence of linear response among extended 
optimal velocity models. Phys Rev E, 69, 017103-1-3.

[28] Ge, H X., Dai, S. Q., Dong L. Y., \& Xue, Y. (2004). Stabilization effect of traffic flow in an extended car-following model based on an intelligent transportation system application. Phys Rev E, 70, 066134-1-6.

[29] Ge, H X., Dai, S. Q., \& Dong L. Y. (2006). An extended car-following model based on intelligent transportation system application. Phys A, 365, 543-548.

[30] Li, Z. P., \& Liu, Y. C. (2006). Analysis of stability and density waves of traffic flow model in an ITS environment. Eur Phys J B, 53, 367-374.

[31] Komatsu, T., \& Sasa, S. (1995). Kink soliton characterizing traffic congestion. Phys Rev E, 52, 5574-5582.

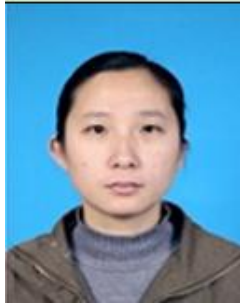

Lei Yu was born in 1980. She received the B.S. degree from Henan Univ. in 2002, the M.S. degree and Ph.D. degree from Northwestern Polytechnical Univ. in 2006 and 2010, respectively. Her research interests are traffic flow modeling and simulation.
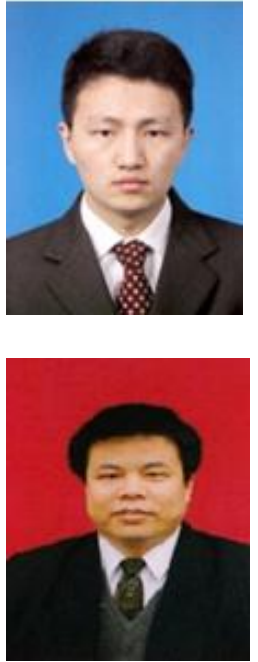

Bingchang Zhou was born in 1981. He received the M.S. degree and Ph.D. degree from Northwestern Polytechnical Univ. in 2005 and 2009, respectively. His research interest is onlinear dynamics.
Zhongke Shi was born in 1956. He received the B.S. degree from Northwestern Polytechnical Univ. in 1981 and his M.S. degree and Ph.D. degree from the same school. His research interests are traffic control and control theory. 